\title{
An Approach to Enterprise Application Integration Based on Ontology Semantic Description
}

\author{
Lu Liu, Deyu Kong, Yi Li and Zhe Liu \\ School of Economics \& Management, Beihang University, Beijing 100083, P.R. China \\ liulu@buaa.edu.cn kongdeyu@buaa.edu.cn
}

\begin{abstract}
This paper systematically provides an enterprise application integration (EAI) approach for the complex product design industry in which the application integration has long been difficult. In addition, the complex product's data sources are distributed in different enterprise application systems which resulted in the design ontology's heterogeneousness. Similarly, the differences in enterprise application systems bring about the heterogeneousness of the application ontology. Based on an actual project, this paper studies the nonobjective characteristic of the heterogeneous design ontology and heterogeneous application ontology in the complex product design industry, and establishes the common semantic description models for the two types of ontology respectively. In the actual project, an information system is developed and has been successfully applied to an enterprise.
\end{abstract}

Keywords: Enterprise application integration (EAI), Ontology, Web services

\section{INTRODUCTION}

With the development of information technology, the prevalence of enterprise information systems brings about higher efficiency as well as some new problems to the enterprises. The implementations of a variety of enterprise information systems need the support of the enterprise's existing resources and applications and consequently bring new challenges to the enterprise. Enterprise Application Integration (EAI) was brought under these conditions and it has changed with the continuously development of Web Services. The rise of XML and Web Services provides a new direction for EAI [1].

EAI is defined as the integration of the process, software, hardware and standards of different application systems, making the systems operate seamlessly in the bound of an enterprise. EAI not only supports the integration of the internal application systems but it also supports the integration of different enterprises' application systems [2].The models of EAI includes three layers: the integration in the representation level, the integration in the data level, the integration in the function level.

There are a great deal of data stored in different ways in the enterprise due to the different stages of information systems implementation, the technologies used by the information systems and some human factors. These data compose the heterogeneous

Please use the following format when citing this chapter:

Liu, L., Kong, D., Li, Y., Liu, Z., 2007, in IFIP International Federation for Information Processing, Volume 255, Research and Practical Issues of Enterprise Information Systems II Volume 2, eds. L. Xu, Tjoa A., Chaudhry S. (Boston: Springer), pp. 977-982. 
data sources. The heterogeneousness of the enterprise's data sources is exhibited as follows:

1. The different data sources use different terms to represent the same concept.

2. The same term expresses different meanings in different data sources.

3. There are various relations between the concepts in different data sources, but due to the autonomy of the distributed data sources, the implicit relations are not been exhibited (e.g. the inconsistency of the measurement).

Ontology describes the meanings of concepts accurately, describes the implicit relations between the concepts and acquires the implicit relations through logical reasoning. It has powerful capabilities to represent the semantics of the concepts and acquire knowledge. Hence, Ontology is used to solve the problem of heterogeneous data [3]. This paper systematically provides an enterprise application integration (EAI) approach for the complex product design industry in which the application integration has long been difficult. A Complex product is a type of product with complex consumer demands, complex components, complex manufacturing process, complex experiment and maintenance process, complex project management and complex work environment, such as air crafts, airplanes, automobiles, and ships, complex mechanical and electronic products and so on.

\section{EAI BASED ON ONTOLOGY SEMANTIC DESCRIPTION}

Ontology is a concept originally coming from philosophy, and it describes the objective existence of a system and concerns the abstract nature of the objective reality. In the artificial intelligence field, the first definition of Ontology was given by Neches et al. In their literature [4], the Ontology was defined as nomenclature, relationships and the definition that use these terms and relations constitute the vocabulary extension of these rules in related fields. In the artificial intelligence field, Studer's definition about Ontology was generally accepted. Knowledge ontology is a clear formalization specification of the shared conceptual model. This definition includes four levels of implications [5].Based on Figure 1, the current research of Semantic Web mainly focuses on ontology layer. The precise meaning of Ontology is achieved by the strict definition of concepts and the relationship between the concepts. Ontology is a clear conceptual model of the specification [6]. So in the field of Semantic Web, ontology lies in a very important position and is the basis to solve Web information sharing and exchange in semantic level. Ontology technology and other artificial intelligence technologies rely on the knowledge representation and reasoning technology, but ontology-based system is different from the knowledge base systems, expert systems and the main technology (Agent Technology) system significantly in the following aspect. Ontology description puts more emphasis on data sharing. Ontology expresses the general sharing knowledge but not some personal data. Currently, the common ontology languages are OIL [7], DAML+OIL [8] and OWL. 
An Approach to Enterprise Application Integration Based on Ontology Semantic

Description 979

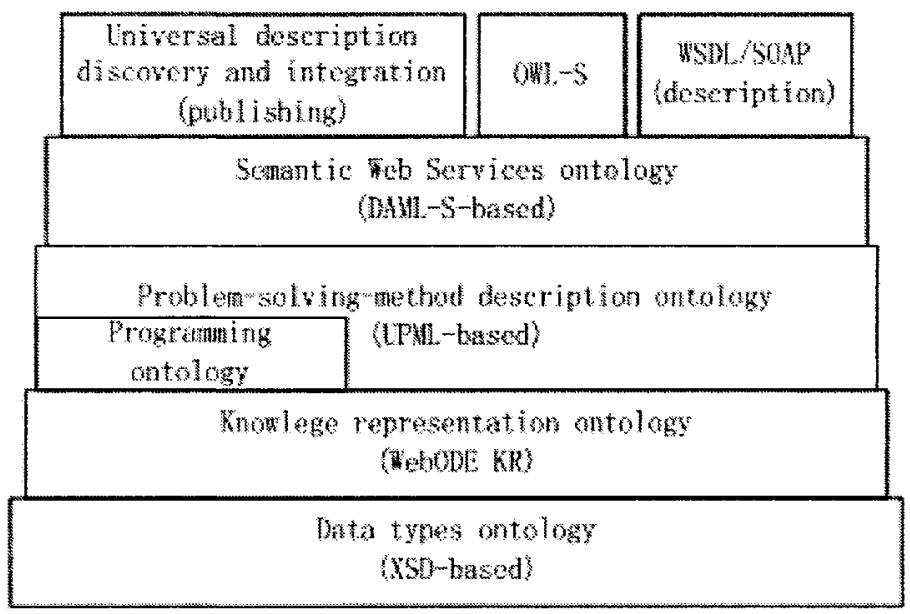

Figure 1. The Framework for Semantic Web Services based on Ontology

\section{DESIGN OF EAI AND REALIZATION IN AN ENTERPRISE}

The goal of data integration of heterogeneous data sources is to provide integrated, unified, secure and easy access to information inquiries, data mining and high-level decision-making for enterprise application systems. To meet the requirements, the integrated data must have a unified data model with integrity, consistency and security. Ontology is the basis of concept share, and it helps us to explain the data consistency [8]. We adopt the global ontology base to solve the problem of heterogeneous data sources of enterprise application systems. The framework diagram is shown in Figure 2. The functions of every layer are explained as follows:

1. The Information source layer is the enterprise data provider to provide data in various storage formats from various enterprise application systems.

2. The Middleware layer composed of wrapper and inquiries engine converts the heterogeneous data into XML or converts XML into heterogeneous data by constructing wrappers for the different data sources so as to establish a two-way mapping.

3. The XML interface layer is responsible for the communication between the users.

4. The Data integration layer is to create a global ontology to change the heterogeneous data packed and stored in different wrappers into a unified data format in order to eliminate the differences between the syntax and semantics.

5. The Information services layer is to apply the integrated data with integrity, consistency and safety.

XXX.Electric Co., Ltd. was founded in 1958, and it has become one of the largest companies specializing in manufacturing large electronic products. With the rapid increase of the demand of the products, how to optimize and integrate the original 
product design flow has become an important problem. After an analysis of the design flow of the products, some problems were found in the field of product design.

The calculating software used for designing and calculating the parameters of the products is independent. The designer must write a great deal of data at each step for the calculating software. The outputs of the calculating software are txt files and graphics files. There is no comparison, filtering and optimization between the outputs.



Figure 2. The Framework based on the Global Ontology Base

Based on the above problems, the Extensible Electrical Product Design Integration Environment is designed. Figure 3 shows the problem-solving framework of the Extensible Electrical Product Design Integration Environment. The framework uses ontology-based scalable module definition format language (SMDFL language), relevant data storage and Web services to realize the extensible model of the electrical product integration design platform. SMDFL supports the description, storage, retrieval and validation of complex data, provides scalability for the calculation process, supports the optimization of data retrieval and helps the data storage model to support generic application program interface (API). The effective integration of SMDFL engines and data storage can support metadata management, derivative data management, database management, modern intelligent graphical user interface, the tools of scientific visualization and analysis and the effective realization of case-based reasoning system. Electrical Product design integration environment based on the service-oriented framework has the characteristics of high scalability and loose 
coupling. Electrical Product design integration environment provides a unified graphical user interface to facilitate the integration of various software modules. By using the various functions of electrical product design integration environment, designers can achieve the efficient storage, retrieval, analysis and reuse of the large amount of electrical product design cases in the design lab to support the Decision Support System based on the case-based reasoning [9].

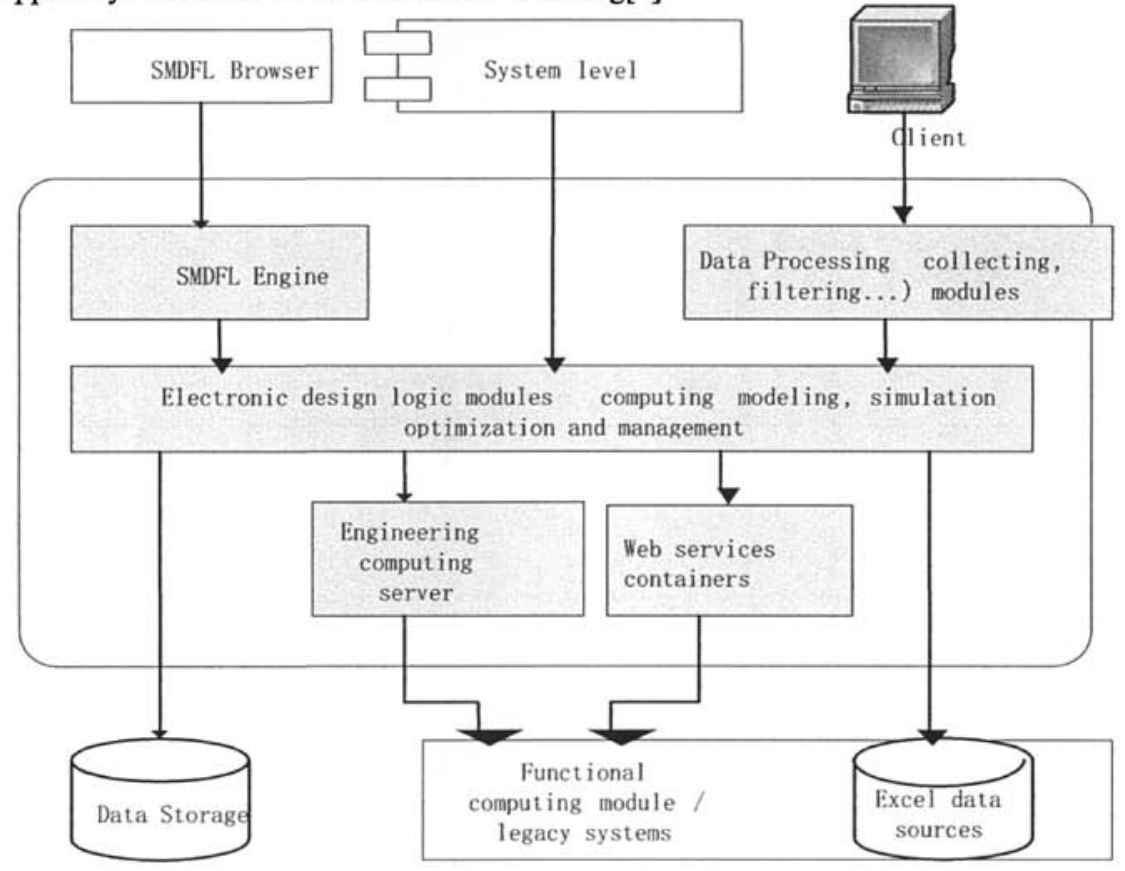

Figure 3. The Framework of Electrical Product Design Integration Environment

\section{CONCLUSIONS}

This paper proposes a method of enterprise heterogeneous data integration based on ontology, which can solve the problem of Information Island and process the heterogeneous data sources in enterprise application integration. This method not only saves the human resources and financial resources of the enterprise, but also ensures the update of enterprise application systems. The author and his project team members designed an EAI platform and successfully applied to an enterprise on the basis of the ontology-based EAI method. 


\section{ACKNOWLEDGEMENTS}

Funding for this research is supported by the National Natural Science Foundation of China under Grant No.70671007 and the PhD Program Foundation of Education Ministry of China under Contract No. 20040006023.

\section{REFERENCES}

1. T. Payne and O. Lassila, Semantic Web Services, IEEE Intelligent Systems. Volume 19, Number 4, pp.14-15, (2004).

2. A. Gómez-Pérez and R. González-Cabero, A Framework for Designing and Composing Semantic Web Services, IEEE Intelligent Systems. Volume 19, Number 4, pp.24-31, (2004).

3. H. Wu and G. Xing, Research on technology of information integration based on ontology, Computer Applications. Volume 25, Number 2, pp.456-458, (2005).

4. R. Neches, R.E. Fikes, and T.R. Gruber, Enabling Technology for Knowledge Sharing, $A I$ Magazine. Volume 12, Number 3, pp.36-56, (1991).

5. R. Studer, V.R. Benjamins, and D. Fensel, Knowledge Engineering, Principles and Methods, Data and Knowledge Engineering. Volume 25, Number1-2, pp.161-197, (1998).

6. M.L. Conway, Towards the principled engineering of knowledge, AI Magazine. Volume 3, Number 3, pp.4-16, (1982).

7. H. Roth, D.A. Waterman, and D.B. Lenat, Building Expert Systems (Reading, AddisonWesley: MA, 1983).

8. R. Neches, R.E. Fikes, and T.R. Gnuber, Enabling Technology for Knowledge Sharing. $A I$ Magazine. Volume 12, Number 3, pp.36-56, (1991).

9. Y. Li, A Scalable Module Definition Format Language Based on XML and its Application. Master Thesis, School of Economics \&Management Beihang University (2007). 